\title{
Avatars de la dénomination et de la désignation dans les fictions persanes : construction d'un point de vue ironique
}

\author{
Frédéric Calas \\ Université de Clermont-Ferrand (EA1002 CELIS) \\ calasfrederic@wanadoo.fr
}

L'objet de cette étude est de cerner des phénomènes d'hétérogénéité énonciative qui se manifestent dans des séquences définitionnelles, abritant des marqueurs de glose, c'est-à-dire des séquences qui supposent un retour sur le dit et le dire, une retouche corrective. Le corpus retenu pour conduire cette enquête est constitué de deux romans par lettres apparentés : Les Lettres persanes ${ }^{1}$ de Montesquieu (1721) et Les Lettres d'une péruvienne de Mme de Graffigny (1747). En effet, dans les fictions persanes ${ }^{2}$, un abondant lexique métalinguistique est destiné à dénoncer une déficience de la désignation ou de la dénomination due au statut d'étranger de certains explorateurs. Sous la feinte incapacité à nommer une réalité familière aux lecteurs français, les écrivains ne cessent de questionner non seulement le rapport des mots aux choses, mais aussi l'essence même des choses, qui, dans les domaines religieux, politique ou social, leur semble totalement dysfonctionner. Ainsi, dans la rencontre d'un étranger, qu'il soit Inca, Indien, Persan, et d'un Français naissent des points d'incompatibilité issus de deux univers discursifs différents.

Comme l'a fait remarquer Roland Barthes (1984 : 118), c'est la « synonymie qui permet au langage de se diviser ». Dans les fictions persanes, il se fissure pour dire tout à la fois la division du pouvoir et les abus de pouvoir. La synonymie s'instaure alors sur le mode de la traduction d'un terme officiel français par un terme approximatif supposé appartenir à la langue perse ou inca; en réalité, il s'agit d'un terme métaphorique également français ${ }^{3}$. L'altérité se fait alors strictement linguistique dans le mouvement de métaphore - qui est bien, selon l'étymologie, un transfert ${ }^{4}$, un déplacement -, au sein de la langue française. La fiction de l'autre, de l'étranger, devient un dispositif rhétorique efficace dans la mise en place d'un questionnement sérieux sur les fondements du pouvoir. En s'élaborant contre lui, contre la doxa, les mots des Persans ou des Incas apparaissent alors dans tout leur éclat para-doxal.

\section{Marqueurs de glose : de l'entreprise définitoire à l'apprentissage linguistique}

L'hétérogénéité de certains termes se trouve soulignée par le choix de marqueurs de glose $\mathrm{e}^{5}$ introduisant une distance métalinguistique dans le système de désignation des êtres et des choses, que le jeu typographique accentue encore.

L'entreprise de glose des Persans part d'une défaillance de la dénomination, en mettant en relation ce qui passe pour deux systèmes ${ }^{6}$ : la périphrase qui décrit sur le mode de l'à-peu-près la chose vue et le nom conventionnel pour la désigner en français.

\subsection{Un marqueur typographique d'hétérogénéité montrée : I'italique}

Grâce à l'italique, le point d'articulation du monde étranger et de l'univers français devient palpable et apparaît dans toute sa complexité, exactement au point crucial où il ne va plus de soi. C'est une efficace représentation du choc de ces mondes, tant sur le plan linguistique que sur le plan des idées, qui s'exhibe sur le mode de l'hétérogénéité montrée ${ }^{7}$, puisque l'italique rend le segment perçu comme «autre» visible, palpable, et de ce fait montré du doigt. Dans l'entreprise de commentaire et de glose, on remarque que cette opération cognitive met en relation deux univers, celui du mot (ou de la séquence support) et son commentaire. 
L'italique souligne pour le lecteur le terme pivot et problématique, et se greffe sur l'élément dont on teste la légitimité, en dénonçant sa non-pertinence linguistique pour révéler la source de sa facticité. La glose, marquée par l'italique, indique une opacité, là où l'on croyait trouver une transparence. Elle se dote, en raison de sa fréquence, d'un pouvoir critique efficace. L'italique occupe un pôle stratégique dans ce dispositif, en désignant très exactement le point de tangence entre le monde oriental et le monde occidental. C'est un carrefour où convergent deux lignes interprétatives, du choc desquelles jaillit l'interrogation, perfide mais pertinente, des voyageurs attentifs.

Dans la veine des fictions persanes, cette mise en relation ne va jamais de soi et sert de correspondant microstructural et métalinguistique, à la structure générale des œuvres elles-mêmes, dont le but est de confronter deux mondes : la France (présentée comme ce qui est inconnu) et le pays d'origine des voyageurs (Perse, Pérou, Canada, etc.), forme locale d'une sémiotique générale dans une iconisation du conflit.

Dans les Lettres persanes, le terme mis en italique est toujours un mot français, que les Persans découvrent et qui devient la source de leur étonnement. Ce terme unique (les éléments mis en italique ne vont jamais au-delà d'un syntagme nominal avec adjectif ${ }^{8}$ ) est introduit dans les deux textes examinés par deux verbes, « appeler ${ }^{9} »$ et $«$ nommer $^{10} »$.

Tout le peuple s'assemble sur la fin de l'après-dînée, et va jouer une espèce de scène que j'ai entendu appeler comédie. Le grand mouvement est sur une estrade, qu'on nomme le théâtre. Aux deux côtés, on voit, dans de petits réduits qu'on nomme loges, des hommes et des femmes qui jouent ensemble des scènes muettes, à peu près comme celles qui sont en usage en notre Perse. (L. XXVIII)

Quoique les Français parlent beaucoup, il y a cependant parmi eux une espèce de dervis taciturnes qu'on appelle chartreux. On dit qu'ils se coupent la langue en entrant dans le couvent, et on souhaiterait fort que tous les autres dervis se retranchassent de même tout ce que leur profession leur rend inutile. (L. LXXXII)

De cette passion générale que la nation française a pour la gloire, il s'est formé dans l'esprit des particuliers un certain je ne sais quoi, qu'on appelle point d'honneur. C'est proprement le caractère de chaque profession; mais il est plus marqué chez les gens de guerre, et c'est le point d'honneur par excellence. Il me serait bien difficile de te faire sentir ce que c'est: car nous n'en avons point précisément d'idée. (L. XC)

\subsection{Deux marqueurs métalinguistiques : les verbes appeler et nommer}

Ces lexèmes verbaux relèvent de la même démarche métalinguistique ${ }^{11}$ donnant une définition, plus exactement une dénomination, à une chose ou à une personne. Cette tentative définitionnelle se présente sous la forme d'une périphrase, dans la mesure où les Persans rencontrent des difficultés pour affecter le mot juste à la réalité qu'ils découvrent. Cette démarche nous permet de rappeler la question qui fut débattue par Cratyle et Hermogène dans le célèbre dialogue de Platon, lorsqu'ils s'interrogent sur la justesse des noms. Selon Jürgen Trabant (1992: 49) «Voilà pourquoi Platon introduit dès le début du dialogue son alternative à ce problème de l'image, sa célèbre formule selon laquelle les mots seraient des outils (órganon) permettant de distinguer l'essence et d'enseigner (Crat. 388b). Cette caractérisation des mots comme outils, et de la parole comme tekhnê, comme activité artisanale, comme force productive active de l'homme, confère au langage une nouvelle qualité. Un outil que l'on peut utiliser pour faire quelque chose est un concept plus actif en regard du concept essentiellement passif du nom adamique, du juste mot-copie magique, de l'image qui ne fait que doubler la création. » La démarche attribuée par Montesquieu à ses personnages semble justement aller dans le sens d'une recherche d'activité, ou pour le moins d'une interrogation, sur les relations liant le mot à la chose. 
Les Persans sont alors contraints de proposer une définition fondée sur une description ${ }^{12}$. L'avantage de la périphrase, et surtout de celles des Persans qui ont un air naï, est d'écarter les représentations toutes faites liées au vocable habituel, et à faire surgir des propriétés spécifiques, auxquelles le lecteur, surpris, ne s'attendait pas.

Les emplois repérés dans les textes relèvent tous d'une dimension métalinguistique, puisque, selon le $T L F^{13}$, dans ces constructions, appeler, par exemple, a le sens de «donner un nom». Grâce à la réflexivité du langage, c'est au départ la relation de signe à signe qui est interrogée, mais elle implique que soit aussi examinée la relation de signe à personne, ou de signe à chose. En effet, pour accéder aux choses, il faut connaître les mots. Comme l'ont montré les philosophes, nous ne pouvons séparer la formation et la conception de nos pensées de leur formulation. Or, cette formulation suppose une langue que nous n'avons pas inventée ${ }^{14}$ et qui a une longue histoire. Les analystes du discours et les sémanticiens retrouvent là le discours des philosophes et des logiciens. La langue dans laquelle je m'exprime est imprégnée de dialogisme et de stéréotypie. C'est la raison pour laquelle ces séquences définitionnelles et métalinguistiques sont relativement nombreuses dans les œuvres examinées.

Ces séquences $\mathrm{X}$ appelle $\mathrm{Y} \mathrm{Z}, \mathrm{X}$ nomme $\mathrm{Y} \mathrm{Z}$ ont la particularité d'attirer l'attention sur les deux signes mis en présence, et non seulement sur l'un d'entre eux. Dans un phénomène de miroir, c'est la pertinence, déclinée à trois niveaux, qui se trouve mise en question : pertinence de la relation de signe à chose, de signe à personne, et de signe à signe. Grâce au système de glose, c'est tout à la fois la non-coïncidence entre les mots et les choses qui est mise au jour, et la non-coïncidence des mots avec eux-mêmes. Ces séquences développent non seulement une activité métalinguistique, mais déroulent aussi une activité énonciative complexe ${ }^{15}$, dans laquelle l'énonciateur vise le monde à travers le déploiement de deux signes $\mathrm{X}$ et $\mathrm{Y}$, lequel résiste en quelque sorte à l'effacement de la transparence, ce qui génère alors une tension entre $\mathrm{X}$ et $\mathrm{Y}$, au détriment de $\mathrm{Y}$.

Le moule syntaxique répété à intervalles réguliers impose une isotopie dont la continuité énonciative sert de pierre de touche à la critique amusée de Montesquieu. Les constructions qui accueillent ces deux verbes sont toutes bâties sur le même patron syntaxique. C'est dans la relation que construisent les verbes de dénomination qu'apparaît le hiatus. En effet, « le langage de l'autre est perçu selon les arêtes les plus vives de son altérité » (Barthes, 1984 : 118). Le verbe permet de rapprocher pour les identifier, deux termes $^{16}$, l'un familier aux enquêteurs persans (écrit en caractère romain), l'autre relevant de la nomenclature française (mis en italique).

On vient d'établir une chambre qu'on appelle de justice, parce qu'elle va leur ravir tout leur bien. Ils ne peuvent ni détourner ni cacher leurs effets: car on les oblige de les déclarer au juste, sous peine de la vie. (L. XCVIII)

La répétition de ce patron syntaxique tout au long des Lettres persanes crée un leitmotiv d'une frappante régularité, une sorte de refrain qui permet à l'œil du lecteur de se familiariser avec cette procédure, qu'il décrypte au coup par coup, mais aussi dans la continuité d'une série. Les Persans, comme Zilia, s'arrêtent parce qu'ils butent sur les mots. Ils s'interrogent sur leur pertinence et sur la réalité qu'ils recouvrent. Ils se livrent à une quête du sens propre ou de la pertinence dénominative. Cette recherche débute par l'interrogation linguistique. L'italique de la distanciation critique, en tout cas d'une espèce d'éloignement pour l'observation, signale une faille, une différence incompréhensible et a priori inexplicable. La lecture critique des Persans décèle un écart, riche de sens, et dont il va falloir rechercher la motivation. Les termes mis en présence semblent avoir une existence séparée et opposée. L'amorce de définition fonctionne à rebours et débouche sur le doute. La contradiction éclate dans cette tentative de définition analogique. La construction syntaxique permet à la satire de se développer comme le décrit Georges Benrekassa (1987 : 53) quand il écrit : « (...) la confusion de l'être et du paraître, la perturbation des hiérarchies "naturelles", thèmes qui trouvent ici une autre force parce qu'ils sont liés à l'hypothèse romanesque qui fait le livre (...). » 
Les constructions présentes dans les Lettres persanes et les Lettres d'une Péruvienne s'opèrent par les marqueurs appeler ou nommer, construits soit avec un SN complément d'objet direct et/ou un attribut de l'objet, soit pour le seul verbe appeler dans le cadre d'une construction pronominale ${ }^{17}$. Elles reposent sur des schémas du type $\mathrm{X}$ appelle $\mathrm{Y} \mathrm{Z}$ ou X s'appelle $\mathrm{Y}, \mathrm{X}$ nomme $\mathrm{Y} \mathrm{Z}$. Les constructions en appeler sont plus fréquentes dans les Lettres persanes, notamment la construction pronominale, alors que ce sont les constructions avec le verbe nommer qui dominent dans les Lettres d'une Péruvienne. Dans les deux cas, il s'agit d'une opération de dénomination, qui est censée mettre en relation un signe et une chose, dans le cadre d'une opération référentielle. Or, il faut qu'il y ait eu un acte de dénomination préalable pour que $\mathrm{X}$ réfère à l'objet x. C'est là que les choses se compliquent dans l'univers des fictions persanes.

\section{Polyphonie énonciative dans les séquences de glose : circulation de la parole et univers de croyance}

Dans la schématisation proposée, $X$ est le sujet (au sens grammatical du terme) et introduit l'instance énonciative qui appelle ou qui nomme. Dans chacune des occurrences examinées, quelqu'un attribue un nom à quelque chose. Un phénomène de polyphonie énonciative est immédiatement perceptible ici, que les théories d'Oswald Ducrot $(1980,1984)$ permettent de décrire en distinguant utilement entre locuteur et énonciateur. Il s'agit bien de polyphonie, dans le sens où plusieurs " voix », plus exactement plusieurs sources énonciatives, se trouvent convoquées - et partant, s'expriment -, dans l'espace discursif particulier et resserré des séquences de glose. En effet, le choix de la forme épistolaire des textes examinés fait que ces séquences s'inscrivent dans l'énonciation subjective et personnalisée des personnages étrangers, à savoir Usbek, Rica et Zilia. S'ils sont les locuteurs premiers de l'énonciation de ces séquences, on remarque immédiatement qu'ils ne sont pas les sujets grammaticaux, ni les énonciateurs prenant en charge l'acte de dénomination des faits décrits.

\subsection{Locuteurs et énonciateurs : aux sources de la polyphonie}

Dans les séquences examinées du type : «ce magicien s'appelle le Pape», il convient d'isoler dans un premier temps de l'analyse :

$$
\begin{array}{cl}
\text { Rica }_{L}:\left\{\text { ce magicien }_{\text {e1 }}\right. & \text { s'appelle } \left._{\text {ezarb }} \text { le Pape }\right\} \\
\text { X } & \text { s'appelle } \quad Y
\end{array}
$$

- le locuteur de cette séquence prélevée dans le corps de la lettre XXIV ${ }^{18}$, qui est Rica, adressant son texte à Ibben, lequel occupe le pôle du destinataire. La lettre est écrite de Paris, le 4 de la lune de Rebiab 2, 1712. L'ancrage nynégocentrique est donc parfaitement posé. À la suite de Ducrot (1984), on peut distinguer entre $\mathrm{L}$ « le locuteur en tant que tel », ici Rica, et $\lambda$ « le locuteur en tant qu'être du monde ».

- les énonciateurs, qui sont responsables des actes illocutionnaires et du sens de l'énoncé. C'est là qu'il convient de discerner pour les énoncés définitionnels entre :

$\mathrm{e}_{1}=$ l'énonciateur prenant en charge $\mathrm{X}$ dans la séquence $\mathrm{X}$ s'appelle $\mathrm{Y}$.

$\mathrm{e}_{2}=$ l'énonciateur, qui prend en charge "s'appelle $\mathrm{Y}$ », est le plus souvent anonyme. Ainsi, nous proposons de le noter - arb,

Distinguer $\mathrm{e}_{1}$ de $\mathrm{e}_{2}$ est tout à fait nécessaire si l'on veut observer le phénomène particulier de circulation de la parole qui s'opère au sein des énoncés définitionnels recourant à des marqueurs de glose. En effet, le mouvement de la définition va du connu vers l'inconnu, de la saisie éminemment subjective du locuteur persan, qui est l'énonciateur $\mathrm{e}_{1}$, responsable du choix de $\mathrm{X}$, vers la dénomination linguistique standard de la chose. Le degré de subjectivité de $\mathrm{e}_{1}$ peut se mesurer dans le degré de marquage axiologique de X. On peut déjà dire que, plus $X$ tend vers la métaphore - ou se dilue dans l'indéfini -, plus son coefficient axiologique s'accroît, car les mots et les réalités rapprochés se trouvent alors sur un axe éloigné : « magicien $v s$ pape » ou « un je ne sais quoi vs la grâce ». La polyphonie qui s'instaure entre les différents pôles énonciatifs est cinétique et agonistique, puisqu'elle va créer la rencontre de deux univers de 
croyance totalement divergents : celui des Persans et celui de la doxa française. La pensée, en allant de la subjectivité vers la doxa, dans le cadre d'une proposition prédicative définitionnelle procède à l'envers et en miroir. De là, Montesquieu tire au moins deux effets : l'un stylistique, car l'envers est par essence l'univers du rire, du comique, de l'informe, support libérateur de la satire ; l'autre heuristique, car le miroir est le lieu d'un questionnement de soi et de l'autre, puisqu'il crée un point de rencontre entre deux univers hétérogènes, mais censés exprimer le même.

Rappelons que la glose comme la définition prédicative se rattachent au mécanisme général de la synonymie, et posent chacune un rapport d'équivalence entre $\mathrm{X}$ et $\mathrm{Y}$. Relevant de deux sources énonciatives différentes $\mathrm{e}_{1}$ et $\mathrm{e}_{2}, \mathrm{X}$ et $\mathrm{Y}$ entrent en relation conflictuelle : très exactement s'y réalise ce que Michael Bakhtine (1978) appelle une «interaction». Montesquieu réussit grâce à ces séquences à extérioriser la doxa et à la constituer en discours autonome qui laisse entendre sa voix; c'est par l'intermédiaire de la fiction des voyageurs persans qu'il arrive à dissocier cette voix, le plus souvent difficile à identifier ou à isoler ${ }^{19}$, car elle parle en nous et nous constitue. Procédant à l'envers et de l'extérieur, l'écrivain finit par l'insérer dans un environnement où elle ne correspond plus au discours dominant, et en devenant visible ou audible, elle donne prise à la critique.

\subsection{Le cas du ON-locuteur}

Examinons à présent le pôle $\mathrm{e}_{2}$. Cette source, le plus souvent anonyme, est introduite par le pronom personnel dit indéfini on, qui en devient l'énonciateur, et, qu'à la suite des travaux de Berrendonner (1981 : 58), on peut nommer ON-locuteur, lequel, selon cet auteur, " dénote une doxa anonyme ». Or, l'émergence d'un gommage énonciatif au sein d'une énonciation embrayée génère un flottement, qui se transforme rapidement en tension. Et ce à deux niveaux - l'un énonciatif, l'autre heuristique, et avec au moins deux effets - l'un philosophique, dans un geste de mise à distance, l'autre satirique dans une posture critique.

Toujours selon Berrendonner (1981), et contre l'avis d'Anscombre (2005: 81), « on peut inclure aussi bien que ne pas inclure le locuteur et/ou le destinataire, et ceci reste affaire de conjoncture ». On est typiquement un marqueur de polyphonie, mais qui pose des problèmes particuliers dans la mesure où il est parfois difficile de savoir s'il inclut, ou non, le locuteur et/ou le destinataire. Dans le cas des fictions persanes, il semble assez clair que les séquences de glose examinées se réalisent sur le clivage des sources énonciatives et des univers représentés. On est alors ouvertement et totalement du côté de la source « française », de laquelle d'ailleurs les Persans et leurs destinataires « restés dans notre Perse » s'excluent en se sentant extérieurs ${ }^{20}$. Il renvoie donc à cette doxa anonyme dont parle Berrendonner, et qu'il est justement difficile d'identifier.

Selon Danielle Leeman (1991 : 106), employer on « revient à établir la société humaine tout entière comme agent du discours, [et] rendre universelle la portée de sa parole ». Anscombre ${ }^{21}$, dans sa critique de la théorie de Berrendonner au sujet du ON-locuteur, introduit un outil important qu'il emprunte à Jacqueline Authier-Revuz (1992-1993), celui de «marqueur de modalisation en discours second ». Il est alors intéressant de montrer que dans les séquences du type :

\footnotetext{
Quoique les Français parlent beaucoup, il y a cependant parmi eux une espèce de dervis taciturnes qu' $\underline{\mathbf{o n}}_{1}$ appelle chartreux. $\mathbf{O n}_{2}$ dit qu'ils se coupent la langue en entrant dans le couvent, et on $\mathbf{n}_{3}$ souhaiterait fort que tous les autres dervis se retranchassent de même tout ce que leur profession leur rend inutile. (L. LXXXII)
}

le pronom on, repéré par l'indice 1, sert plus à indiquer la source ou l'origine du discours des autres (ici d'ailleurs le texte précise clairement les Français), dont le locuteur s'exclut, qu'à rapporter ses propos ${ }^{22}$. Dès la deuxième occurrence, repérée par l'indice 2 , la fonction se modifie puisque ce qui était la désignation de la source d'attribution de nom s'ouvre au discours rapporté. Quant à la troisième occurrence, elle est sans doute la plus complexe sur le plan énonciatif. On peut penser qu'il s'agit d'une 
simple reprise de même rang que le $\mathrm{on}_{2}$, ou alors plus vraisemblablement poser une énallage personnelle introduisant un nouvel énonciateur - Montesquieu à peine dissimulé derrière la voix de ses Persans -, car le pronom est alors non plus le sujet d'un verbe dicendi, mais d'un épistémique évaluatif, souligné par le conditionnel. Comme la vulgate linguistique de ces dernières années l'a abondamment montré, le pronom on connaît une souplesse énonciative qui en fait un condensé de polyphonie, à géométrie variable. Ce qui est intéressant d'un point de vue stylistique, c'est que cette propriété énonciative du pronom on introduit une polyphonie, que l'on peut évaluer en termes de dynamisme. Dans l'occurrence extraite des Lettres persanes, il ne suffit pas d'épingler les strates polyphoniques convoquées grâce à la souplesse du pronom on, mais davantage de voir comment s'instaure une circulation de la parole entre les trois pôles, qui revient à l'expression de trois points de vue différents et divergents sur les religieux, ici ceux de l'ordre des Chartreux. Sous le choix d'une forme unique, dans une sorte d'antanaclase externe, Montesquieu fait parler au moins trois voix, qui ne s'accordent pas sur la chose examinée. De cette polyphonie par clivage naît une satire, d'autant plus fine qu'elle pourrait passer inaperçue. Il s'agit de la convocation d'une source anonyme relevant de la doxa, qui devient une « voix », et qui dit bien le discours d'un autre (ou des autres), face à la voix des Persans. Il ne peut s'agir dans le cadre dichotomique construit par Montesquieu d'un phénomène de dialogisme, c'est-à-dire de la présence d'un déjà-dit dans le discours, mais bien d'une tentative délibérée pour dissocier cette part d'implicite, de présupposé, de déjà-dit qui enlise tout discours et la faire entendre, grâce au stratagème du clivage des voix et des univers de croyance, comme une voix identifiable (même si elle demeure anonyme ou collective), à laquelle on reproche la part de sclérose qu'elle véhicule.

Faire abstraction de cette intention générale - appelons-la macrostructurale, reviendrait à dénaturer le sens même des énoncés. "Ce magicien s'appelle le Pape» est une séquence polyphonique où se rencontrent au moins deux voix qui s'expriment (et qui ne sont pas simplement convoquées ou placées dans le même énoncé). Elles disent chacune quelque chose de radicalement différent. Là où la voix anonyme et collective de la doxa désigne une fonction, institutionnalisée et sacralisée (un pape), les Persans identifient une usurpation, un leurre, une imposture (un magicien). Si l'on analyse cette séquence en termes de dialogisme, on ne fera qu'essayer d'y trouver la présence, ou les traces, d'une énonciation autre, qui serait en quelque sorte sous-jacente à celle présente en surface, comme si l'on procédait verticalement. En revanche, en termes de polyphonie, on est amené à traiter les deux voix sur le mode de l'expression, du face-à-face et de la confrontation, dans une saisie plus horizontale, même si les voix sont englobées dans un énoncé unique, produit par une source locutrice L (ici Rica).

Ensuite, dans les séquences de glose à visée définitionnelle, une défaillance affecte alors le système linguistique de la dénomination en langue, et partant, les êtres ou les choses qu'il désigne. Certaines constructions manifestent, par le biais du participe passé passif, un effacement de ce sujet de la désignation (appelé, nommé) ${ }^{23}$. Or, cette source fondue dans l'anonymat, et perdue dans l'histoire de la langue, rend la démarche d'identification et d'explication immédiatement suspecte. Le pronom on, qui apparait comme le sujet le plus fréquent du verbe de dénomination, recouvre une source énonciative française, qui est le «donneur de nom» (Kleiber, 1984: 80). La critique de Montesquieu se tourne également vers cette source énonciative, le plus souvent anonyme et collective, pour dénoncer soit l'absence de pertinence de cette opération préalable ou originaire de nomination, soit les disconvenances innées et acquises, ontologiques ou historicisées.

\subsection{Le cas du "ILS-collectif" $"$}

On ne relève, dans les Lettres d'une Péruvienne, qu'une seule occurrence ${ }^{25} \mathrm{du}$ pronom personnel ils pour désigner la source $\mathrm{e}_{2}$ de dénomination, à savoir le groupe des Français, ce que l'on peut établir par inférence contextuelle ou, comme le propose F. Cornish (1986:133), par « une inférence à un référent discursif latent via un référent discursif existant» : 
même ce qu'ils $\mathrm{s}_{\mathbf{e} 2}$ appellent politesse cache légèrement leurs défauts sous les dehors de la vertu ; mais avec un peu d'attention on ${ }_{\mathrm{e} 3}$ en découvre aussi aisément l'artifice que celui de leurs fausses richesses. (L. XX)

Le choix de cette expression personnalisée, tout en conservant un degré de généralité quasi identique à celui de on, dessine une interaction culturelle plus grande, puisque Zilia identifie la source de dénomination et la dénonce en quelque sorte. On remarquera, de plus, qu'il s'agit d'un emploi particulier, quasi nominal, et non plus endophorique de cet élément, ce qui le rattache à un effet d'anaphore associative; il permet de désigner un groupe d'individus, dont les membres restent anonymes. Cette association, que Georges Kleiber distingue de l'anaphore associative au sens strict, s'opère certes par pontage inférentiel (Clark, 1977), mais la relation va du groupe vers les membres qui le composent. Notons cependant que la relation inférentielle nécessaire à l'identification du référent du pronom collectif est nettement plus discursive qu'encyclopédique ou stéréotypique. En effet, ce n'est que dans la logique de la fiction mise en place par Mme de Graffigny que l'on peut comprendre, dans le macrocontexte de l'œuvre, la référence visée par ils. C'est parce que l'on sait que dans ce roman, l'héroïne est péruvienne et enlevée par des Espagnols, puis recueillie par des Français, que l'ensemble de ses lettres concernent le nouveau monde dans lequel elle évolue: la France. Le pontage inférentiel, tout à fait nécessaire à l'identification du référent, ne repose pas sur une relation stéréotypique a priori ou encyclopédique, mais sur une relation discursive particulière, qui fait que dans cette œuvre s'opposent deux mondes : le Pérou des Incas et la France ${ }^{26}$.

$$
\begin{array}{llll}
\text { ce qu' } & \text { ils }_{\mathrm{e} 2} & \text { appellent } & \text { politesse } \\
\mathrm{X} & \text { ils } & \text { appellent } & \mathrm{Y}
\end{array}
$$

L désigne le locuteur de l'énoncé, ici Zilia, la Péruvienne et $e_{2}$ l'énonciateur, source de la dénomination. Dans le contexte de cette lettre, le choix d'un pronom personnel inscrit plus fortement le clivage des points de vue que ne le ferait le pronom on. En effet, on remarque que le contexte développe une critique du divorce entre l'être et le paraître, qui se glisse, selon Zilia, jusque dans le choix des dénominations. De plus, le pronom on, qui apparaît en clausule de phrase, devient une énallage des deux sources énonciatives, puisqu'il associe le lecteur dans le geste d'analyse critique conduit par l'étrangère : $e_{3}=\mathrm{L}+$ $\mathrm{e}_{4}\left(\mathrm{e}_{4}\right.$ étant le lecteur ou toute personne attentive ayant un sens critique).

C'est donc bien en termes de polyphonie que l'analyse des séquences de glose doit être conduite. S'y révèle une tension née de la circulation d'une parole prélevée sur une doxa et mise en relation prédicative avec une parole vive, jaillie de l'énonciation d'un étranger. À partir des analyses proposées par Oswald Ducrot, nous arrivons à isoler des fragments de discours et à retrouver, en chacun d'eux, la source de leur production. Cette polyphonie resterait simplement statique s'il ne s'agissait que d'un étiquetage à visée typologique. Grâce à la structure définitionnelle et à la glose, les sources énonciatives entrent dans une dynamique d'échange et de confrontation, point d'ancrage des interrogations posées par Montesquieu, où se libère une puissante ironie.

L'ironie détectable dans ces séquences de glose et de définition est de nature polyphonique, dans la mesure où les pôles énonciateurs prenant en charge $\mathrm{X}$ et $\mathrm{Y}$ ne sont pas les mêmes et sont doublement mis à distance : distance dans la structure de glose elle-même, distance dans la nature même de l'ethos de ces énonciateurs, comme on l'a vu supra. De cette tension naît l'ironie polyphonique, qui touche à toutes les composantes de l'acte de parole, dictum et modus, énoncé et énonciation, substance et forme du contenu.

\section{Références bibliographiques}

Amossy, R. (2000). L'Argumentation dans le discours. Paris : Nathan - A. Colin.

Amossy, R. éd. (2002). Pragmatique et analyse des textes. Tel Aviv: Université de Tel-Aviv Press, French Department. 
Anscombre, J.-C. Et Ducrot O. (1988). L'Argumentation dans la langue. Liège: Mardaga, coll. « Philosophie \& Langage ».

Anscombre, J.-C. (2005). «Le ON-locuteur: une entité aux multiples visages ». In Dialogisme et polyphonie, Approches linguistiques, J. Bres, P. P. Haillet, S. Mellet, H. Nølke, L. Rosier (éd.). Bruxelles : De BoeckDuculot, p. 75-94.

Authier-Revuz, J. (1982). «Hétérogénéité montrée et hétérogénéité constitutive : éléments pour une approche de l'autre dans le discours ». DRALV, n²6, p. 91-151.

Authier-Revuz, J. (1992). «Repères dans le champ du discours rapporté ». L'information grammaticale, octobre, $\mathrm{n}^{\circ} 55$, p. 38-42.

Bakhtine, M. [1934] (1978). Esthétique et théorie du roman. Paris, : Gallimard, coll. « Tel ».

Barthes, R. (1984). Le Bruissement de la langue, Essais critiques IV. Paris : Seuil.

Benrékassa, G. (1987). Montesquieu, la liberté et l'histoire. Paris : Le livre de Poche.

Berrendonner, A. (1981). Éléments de pragmatique linguistique. Paris : Minuit, coll. « Propositions ».

Bouverot, D. (2005). «Les marqueurs formés sur appeler d'après le TLFi». In Les Marqueurs de glose, A. Steuckardt et A. Niklas-Salminen (éd.). Aix-en Provence : Publications de l'université de Provence, p. 29-36.

Calas, F. (1995). « Le discours préfaciel comme pré-lecture : les deux pseudo-préfaces des Liaisons dangereuses ». In L’Épreuve du lecteur, livres et lectures dans le roman d'Ancien régime, Peeters, Louvain-Paris, B.I.G. n³1, 1995, p. 436-448.

Calas, F. (1995), «L’imposture épistolaire ou le roman en quête d'auteur ». Revue des Sciences Humaines, n²38, avril-juin, Lille, p. 137-148.

Calas, F. \& Garric, N. 2009). «La définition comme procédé discursif dans les Lettres persanes de Montesquieu (1721)».Publif@rum, n6, http://publifarum.farum.it/ezine_articles.php?id=135.

Charaudeau, P. \& Maingueneau, D., éd. (2002). Dictionnaire d'analyse du discours. Paris : Seuil.

Charolles, M. (1990). «L'anaphore associative. Problèmes de délimitation ». Verbum, XIII, 3, p. 119-148.

Clark, H. (1977). «Bridging ». In P. N. Johnson-Laird et P. C. Wasow (éd), Thinking. Cambridge : C.U.P., p. 411420.

Cornish, F. (1986). Anaphoric relations in English and French. London : Croom Helm.

Cusin-Berche, F. (2003). Les Mots et leurs contextes. Paris : Presses de la Sorbonne Nouvelle.

Ducrot, O. (1980a). Les Mots du discours. Paris : Minuit, coll. « Le sens commun ».

Ducrot, O. (1980b). Les Échelles argumentatives. Paris : Minuit, coll. « Propositions ».

Ducrot, O. (1984). Le Dire et le dit. Paris : Minuit, coll. « Propositions ».

Ducrot, O. (1984a). «Esquisse d'une théorie polyphonique de l'énonciation». In Le Dire et le dit. Paris : Minuit, coll. «Propositions », p. 171-233.

Eco, U. (1994). La Recherche de la langue parfaite. Paris : Seuil, coll. « Faire l'Europe ».

Jaubert, A. (1990). La Lecture pragmatique. Paris : Hachette, coll. « Supérieur ».

Jaubert, A. (2005). « Dialogisme et interaction épistolaire ». In Dialogisme et polyphonie, Approches linguistiques, J. Bres, P. P. Haillet, S. Mellet, H. Nølke, L. Rosier (éd.). Bruxelles : De Boeck-Duculot, p. 215-230.

Kleiber, G. (1984). « Dénomination et relations dénominatives ». Langages, n 76, pp. 77-94.

Kleiber, G. (1992). «Ils ont encore augmenté les impôts ou sur le ils dit collectif». In L. Tasmowski et A. ZribiHertz (éd.). Hommage à Nicolas Ruwet, de la musique à la linguistique. Gent: Communication et Cognition, p. 327-344.

Leeman, D. (1991). « On thème ». Linguisticae Investigationes, XV-1, p. 101-113. 
Moeschler, J. (1985). Argumentation et conversation : éléments pour une analyse pragmatique du discours. Paris : Hatier-Crédif, L.A.L.

Rey-Debove, J. (1978). Le Métalangage. Paris : A. Colin.

Rosier, L. éd. (2002). Faits de Langues, n¹9 (« Le discours rapporté »).

Rosier, L. (2004), « La circulation des discours à la lumière de "l'effacement énonciatif" : l'exemple du discours puriste sur la langue ». Langages, ${ }^{\circ} 156$, p. 65-78.

Steuckardt, A. \& Niklas-Salminen, A. éd (2005). Les Marqueurs de glose. Aix-en-Provence: Publications de l'Université de Provence, coll. «Langue et langage ».

Trabant, J. (1986). Apeliotes oder der Sinn der Sprache. Wilhelm von Humboldts Sprach-Bild. München: Wilhelm Fink Verlag. Version française (1992), Humboldt ou le sens du langage, Liège : Mardaga.

Yaguello, M. (1984). Les Fous du langage : des langues imaginaires et de leurs inventeurs. Paris : Seuil.

${ }^{1}$ Le corpus d'étude est composé des Lettres persanes de Montesquieu, Paris, Garnier, Classiques, éd. critique de P. Vernière, 1975 et des Lettres d'une Péruvienne de Madame de Graffigny, 1983, Paris, Garnier-Flammarion, éd. critique B. Bray et I. Landy-Houillon, p. 237-364.

${ }^{2}$ Le terme «fiction persane» est commode pour isoler un sous-genre thématique au sein de l'espace générique du roman par lettres.

${ }^{3}$ C'est essentiellement vrai pour les Lettres persanes. Il n'y a pas de traduction au sens strict, mais il est constamment fait référence à l'univers de la Perse, à l'aune duquel la France, et les termes français, sont évalués; par exemple: "des hommes et des femmes jouent ensemble des scènes muettes à peu près comme celles qui sont en usage dans notre Perse (L. XXVIII). La préface de l'éditeur avait pris soin de préciser que c'est lui qui traduisait « je ne fais donc que l'office de traducteur : toute ma peine a été de mettre l'ouvrage à nos mœurs » (p. 7). Les Lettres d'une Péruvienne insèrent systématiquement le mot inca dans le corps du texte français et optent pour sa traduction grâce à un système de notes: «Le Cacique m'a donné une China jeune et fort vive ». Une note précise qu'une China est une « servante ou femme de chambre » (L. X, p. 280). Cependant dans les deux textes, ce qui apparaît à la place du mot persan ou inca implicite est bel et bien un terme français qui va être mis en regard d'un autre terme français, celui qui, officiellement, désigne la chose concernée.

${ }^{4}$ On pourrait se poser la question de savoir s'il s'agit là d'un transfert de savoir ou d'un transfert d'ignorance ; dans les deux cas la démarche s'inscrit dans la tradition de la translatio studii et se rattache donc à un geste didactique d'explication ou d'explicitation linguistique, puis encyclopédique.

${ }^{5}$ Nous reprenons le titre du recueil d'articles réunis et édités par A. Steuckardt et A. Niklas-Salminen (2005), Les Marqueurs de glose, en souscrivant aux raisons du choix de cet appellatif contre celui de « connecteur »; voir argumentaire p. 5.

${ }^{6}$ Mme de Graffigny optera pour une position plus littérale, en offrant un aperçu de l'hypolangue inca dans l'espace de glose, face à la langue française.

${ }^{7}$ On se réfèrera ici aux analyses de Jacqueline Authier-Revuz (1982).

${ }^{8}$ Le terme présent en $\mathrm{X}$ est toujours un SN ou l'équivalent d'un SN, puisque l'on trouve une relative substantive dans l'une des occurrences «Ceux qui mettent au jour quelque proposition nouvelle sont d'abord appelés hérétiques », qui constitue le segment le plus long de l'œuvre en position X.

${ }^{9}$ Pour établir le corpus d'étude dans les œuvres sélectionnées, nous ne retenons pas les constructions transitives directes du verbe appeler, du type appeler qqn, dans le sens de «le faire venir». Seuls les emplois de type métalinguistique sont examinés.

${ }^{10}$ On trouve une seule occurrence du verbe « intituler », Lettre XXXV. 


\footnotetext{
${ }^{11}$ Voir les travaux et analyses de J. Rey-Debove (1978: $185 s q$.) sur ces deux verbes.
}

12 Ainsi, il est difficile de déterminer la nature exacte de cette entreprise de glose (ou de traduction) « fictive » en relation explicite avec ce qui s'opère dans un contexte «non fictif ». Pour le passage de X à $\mathrm{Y}$, dans la tradition de la glose homérique, on parvient à distinguer « quatre gestes différents, requis par des oppositions linguistiques différentes: (a) archaïque / actuel; (b) rare / usuel; (c) suffixé / syntagmatisé ; (d) figuré / propre » (Steuckardt et al., 2005 : 8). Dans l'entreprise de Montesquieu, on a affaire plutôt au couple (d) figuré / propre, mais avec une altération de la polarité habituelle au fonctionnement de ce couple, qui est que le figuré est propre au locuteur persan qui le formule, et ne se trouve pas en langue, comme le propre correspondant. La convocation de l'imaginaire est alors toute puissante et libère, par l'effet de choc produit dans le rapprochement inattendu de X et de Y, l'acuité de la satire.

${ }^{13}$ TLF, Trésor de la Langue Française, accès en ligne sur le site : http://atif.atilf.fr/tlf.htm

${ }^{14}$ Pour suivre la fabuleuse histoire de la quête de la langue des origines, des langues parfaites ou imaginaires, voir les travaux de Marina Yaguello (1984) et d'Umberto Eco (1994).

15 Pour décrire ce cas particulier de démarche métalinguistique au sein d'une énonciation complexe, Jacqueline Authier-Revuz (1992, 1995) parle, à la suite de Raymonde Rey-Debove (1978), de « connotation autonymique ». Notons cependant qu'à la différence de ce que pense Jacqueline AuthierRevuz (2003: 74), pour qualifier les séquences ce que $z$ appelle $X$, nous posons l'existence d'un mécanisme de connotation autonymique dans les fictions persanes examinées. En effet dans son analyse débouchant sur le rejet de ces séquences hors du champ de la connotation autonymique, J. Authier-Revuz fait remarquer que le signe n'apparaît pas en usage. Les séquences qu'elle examine ne sont pas semblables à celles de Montesquieu. Comparons en effet, « Ce qu'un militant appelle "la douleur basque" enflamme le centre de la ville [Le Monde, 26-9-84] et « ce magicien s'appelle le pape ». Les séquences forgées par Montesquieu font en fait intervenir deux signes, pour référer à la même chose. Dans le face-àface définitoire dans lequel ils se trouvent pris s'opère une redéfinition, qui passe par l'emploi en mention du deuxième terme. Pris dans les fourches de la séquence, le terme est à la fois soumis à une activité métalinguistique (visant une définition du mot, et une attribution du mot à une chose) et à une activité définitoire (sur le mode de la définition naturelle, qui est une définition de la chose). L'ensemble de ces activités multiples se déroule bien comme le suggère A. Culioli par " étagements », et par un phénomène de «double bind » générant une violente redéfinition de Y par X.

${ }^{16}$ Il est important à ce stade de reprendre la mise au point opérée par G. Kleiber (1984 : 81) entre nomname (« signe qui dénomme les choses de la réalité ») et nom-noun (une valeur grammaticale). En effet, cette distinction est fondamentale dans le cas particulier des fictions persanes, qui font voler en éclats, dans le cadre de l'interrogation conduite par les explorateurs, l'assimilation trop rapidement et trop mécaniquement opérée en langue française entre les deux acceptions, assimilation qui risque de devenir source de confusion et d'erreur, tant sur les mots que sur les choses.

${ }^{17}$ La construction pronominale du verbe appeler réalise un cas de pronominal à sens passif permettant un gommage de l'actant désignateur : ainsi, dans une séquence du type « ce magicien s'appelle le pape », le sens du verbe devient : « est appelé le pape par X ». Un X, qui, justement, n'est pas explicitement désigné par le texte. En recourant aux analyses proposées par la Grammaire générative, on dirait qu'il y a là un indice impersonnel noté - arb pour marquer son degré de généralité. La question est alors de savoir qui appelle X Y, quelle est la part d'arbitraire de la désignation et la portée critique du gommage de l'actant support de l'acte originel de la désignation. Ce phénomène de gommage de l'actant provoque un déficit informatif, qui prend une portée critique particulière dans le cadre particulier de ce discours, et non dans celui de la langue. 
18 C'est la première lettre écrite de Paris, qui marque un seuil interne important dans l'œuvre (l'achèvement du long voyage d'Ispahan à Paris, Lettres I à XXIII), et qui témoigne des premières réactions exprimées sur la France.

${ }^{19}$ Nous rejoignons l'analyse de Ruth Amossy (2005 : 66) : «Que le déjà-dit et le déjà-su s'inscrivent nécessairement dans la langue ne signifie pas qu'ils constituent un point de vue attribuable à un énonciateur : ils ne deviennent une voix que s’ils sont exhibés et donnés à entendre comme le discours de l'autre ».

${ }^{20}$ Nous trouvons une occurrence offrant une structure voisine de celle des séquences de glose et ayant, pour sujet et pour énonciateur, un on-locuteur, qui ne peut inclure les Persans (on parle de X) : « On parle toujours ici de la Constitution. J'entrai l'autre jour dans une maison où je vis d'abord un gros homme avec un teint vermeil, qui disait d'une voix forte: "J'ai donné mon mandement; je n'irai point répondre à tout ce que vous dites; mais lisez-le, ce mandement, et vous verrez que j'y ai résolu tous vos doutes. J'ai bien sué pour le faire, dit-il en portant la main sur le front: j'ai eu besoin de toute ma doctrine, et il m'a fallu lire bien des auteurs latins. - Je le crois, dit un homme qui se trouva là : car c'est un bel ouvrage, et je défierais bien ce jésuite qui vient si souvent vous voir d'en faire un meilleur. - Lisez-le donc, reprit-il, et vous serez plus instruit sur ces matières dans un quart d'heure que si je vous en avais parlé toute la journée." » (L. CI) Cette séquence offre surtout un montage discursif et argumentatif des plus intéressants. En effet, la source énonciative (on) s'inscrit dans une communauté de laquelle les Persans se trouvent exclus. La séquence qui suit (qui n'est pas ici à proprement parler autonymique « la Constitution ») est glosée par un exemple, indexé, lui, à une source énonciative individualisée - Rica. La reformulation se réalise par un exemple, ce que Françoise Cusin-Berche (2003 : 152) appelle « une reformulation métonymique, c'est-àdire la reprise sous forme d'exemple ».

${ }^{21}$ Il développe également une thèse intéressante en montrant que les cas de ON-locuteur se rattachent à différentes communautés linguistiques. Ce qui est aussi vrai, d'ailleurs, pour les occurrences des Lettres persanes, les Persans mis en scène et les Français n'appartenant pas à la même communauté linguistique ; mais c'est cependant une même doxa qui est convoquée comme référent du ON-locuteur, et non plusieurs sources énonciatives.

${ }^{22}$ Nous pouvons reprendre ici une analyse conduite sur l'emploi particulier de ce on par Françoise CusinBerche (2003 : 148), qui en observe l'usage dans des manuels didactiques destinés à un public de jeunes apprenants. Selon elle, on assiste à « un coup de force discursif, reposant sur l'usage du on suivi du présent de l'indicatif. L'apprenant au moment de la lecture de l'énoncé est exclu de cet indéfini, puisqu'il ignorait jusque là l'appellation ou le concept dont il est question et ne pouvait par conséquent faire partie des usagers du terme ». C'est ce que Montesquieu présente par le biais de personnages placés en situation d'apprentissage et d'accès aux connaissances. Contrairement aux manuels de didactique, le but visé par l'écrivain n'est pas de faire accéder les Persans à un nouvel usage, mais, au contraire, de conduire le lectorat français à réfléchir sur ces questions d'appartenance (ou d'exclusion) à la scène cognitive communément partagée.

23 «Me voici, mon cher Aza, dans une ville nommée Paris : c'est le terme de notre voyage ; mais, selon les apparences, ce ne sera pas celui de mes chagrins ». (L. XIII)

${ }^{24}$ G. Kleiber (1992).

${ }^{25}$ Lors de l'établissement du corpus, nous rencontrons une autre occurrence dans laquelle apparaît un pronom personnel pluriel, ils : «En achevant ces mots nous retournâmes dans le temple du Soleil : c'est ainsi qu'ils nommèrent le merveilleux cabinet. J'eus enfin la liberté de parler» (L. XXXV). Il s'agit ici d'un anaphorique standard, qui reprend, par anaphore associative inférentielle mais « fidèle », le groupe restreint des acteurs de cette scène, à savoir Déterville, sa sœur Céline et le mari de cette dernière, qui, par un acte de baptême, appellent le pavillon qu'ils viennent d'offrir à Zilia « un temple du Soleil », en référence à son origine inca et à la restitution de ses biens, dérobés par les Espagnols et longtemps 
conservés par les Français. Il ne s'agit donc pas d'un cas de ils-collectif au sens strict de cette dénomination par G. Kleiber, mais d'un avatar en quelque sorte, nettement orienté vers un groupe restreint mais non anonyme, dans le cadre de cette œuvre.

${ }^{26}$ L'on rejoint ainsi l'analyse de Michel Charolles (1990 : 133) : « Le discours génère aussi [...] son propre cadre associatif en promouvant des relations inédites, conjoncturelles, qui peuvent n'avoir qu'une validité occasionnelle, mais dont la reconnaissance s'impose au récepteur du fait de la présomption de cohérence qui préside à son interprétation ». 